\title{
A Class of Double Coset Cayley Digraphs Induced by Quasigroups
}

\author{
Anil Kumar V. (Corresponding author) \\ Department of Mathematics, University of Calicut Malappuram \\ Kerala 673 635, India \\ E-mail: anilashwin2003@yahoo.com \\ Shyama M. P. \\ Department of Mathematics, University of Calicut Malappuram \\ Kerala 673 635, India \\ E-mail: shyama.manikandan@gmail.com
}

Received: November 4, 2011 Accepted: November 24, 2011 Published: April 1, 2012

doi:10.5539/jmr.v4n2p128 URL: http://dx.doi.org/10.5539/jmr.v4n2p128

\begin{abstract}
In this paper we introduce a new class of double coset Cayley digraphs induced by quasigroups. These graphs can be considered as the generalization of Double Coset Cayley Digraphs induced by loops. Moreover, various graph properties are expressed in terms of algebraic properties. This did not attract much attention in the literature.
\end{abstract}

Keywords: Cayley digraph, Double coset cayley di-graph, Vertex-transitive graph, Quasigroup

AMS Subject Classification Number (2000): 05C25

\section{Introduction}

A binary relation on a set $V$ is a subset $E$ of $V \times V$. A digraph is a pair $(V, E)$ where $V$ is a non-empty set (called vertex set) and $E$ is a binary relation on $V$. The elements of $E$ are the edges of the digraph. An edge of the form $(x, x)$ is called a loop. A walk in a di-graph is an alternating sequence $W=x_{0}, e_{0}, x_{1}, e_{1}, \ldots, x_{k-1}, e_{k-1}, x_{k}$ where $x_{i}$ are the vertices and $e_{i}$ is an edge from $x_{i}$ to $x_{i+1}$. A walk $W=x_{0}, e_{0}, x_{1}, e_{1}, \ldots, x_{k-1}, e_{k-1}, x_{k}$ is called a path, if all the vertices $x_{0}, x_{1}, \ldots, x_{k}$ are distinct. We will use the notation $\left(x_{0}, x_{1}, x_{2}, \ldots, x_{k}\right)$ for the path $W=x_{0}, e_{0}, x_{1}, e_{1}, \ldots, x_{k-1}, e_{k-1}, x_{k}$. A digraph $(V, E)$ is called (i) trivial if $E=\emptyset$, (ii) reflexive if each vertex has a loop, (iii) symmetric if $E=E^{-1}$, (iv) transitive if $E \circ E \subseteq E$, (v) a hasse diagram if for every positive integer $n \geq 2$ and every $v_{0}, v_{1}, \ldots, v_{n}$ of $V,\left(v_{i}, v_{i+1}\right) \in E$ for all $i=0,1,2, \ldots, n-1$, implies $\left(v_{0}, v_{n}\right) \notin E$, (vi) complete if $E=V \times V$, (vii) connected (some would say strongly connected) if $v$ is connected to $u$ for all $u, v \in V$, (viii) locally connected iff for every pair of vertices $u, v \in V$ there is a path from $v$ to $u$ whenever there is a path from $u$ to $v$, (ix) semi connected for every pair of vertices $u, v$, there is a path from $u$ to $v$ or a path from $v$ to $v$, (x) vertex-transitive if, given any two vertices $a$ and $b$ of $V$, there is some graph automorphism $f: V \rightarrow V$ such that $f(a)=b$, that is, a digraph is vertex-transitive if its automorphism group acts transitively upon its vertices (C. Godsil \& R. Gordon, 2001). Whenever the word graph is used in this paper it will be referring to a digraph unless otherwise stated.

A non-empty set $G$, together with a mapping $*: G \times G \longrightarrow G$ is called a groupoid. The mapping $*$ is called a binary operation on the set $G$. If $a, b \in G$, we use the symbol $a b$ to denote $*(a, b)$. A groupoid $(G, *)$ is called a quasigroup, if for every $a, b \in G$, the equations, $a x=b$ and $y a=b$ are uniquely solvable in $G$ (H. B. Richard, 1971). This implies both left and right cancelation laws.

Let $G$ be a group and let $S$ be a subset of G. The Cayley di-graph of $G$ with respect to $S$ is defined as the di-graph $X=(G, E)$, where $E$ is a subset of $G \times G$, such that $(x, y) \in E$ if and only if $x^{-1} y \in S$. The cayley graph of $G$ with respect to $S$ is denoted by $\operatorname{Cay}(G, S)$. The subset $S$ is called the connection set of $X$. That is, Cayley di-graph Cay $(G, S)$ has as its vertex-set and edge-set, respectively, $V=G$ and $E=\{(x, y): y=x z$ for some $z \in S\}$ (E. Dobson, 2006, p. 105-150).

Theorem 1.1 Let $G$ be a group and let $A$ and $B$ be subgroups of $G$. Let $D$ and $D^{*}$ be subsets of $G$. Let $G /[A, B]$ denote the collection of all double cosets of $A$ and $B$ in $G$. Let

$$
R_{D, D^{*}}=\left\{(A x B, A y B): y=z_{1} x z_{2} \text { for some } z_{1} \in A D A, z_{2} \in B D^{*} B\right\}
$$

Then $\left(G /[A, B], R_{D, D^{*}}\right)$ is a graph.

The graph defined in theorem 1.1 is called the double coset cayley digraph induced by groups (K. V. Anil, 2011, p. 747 - 
753). Observe that if $A=B=D=\{1\}$, then double coset cayley digraph $\left(G /[A, B], R_{D, D^{*}}\right)$ reduces to a Cayley di-graph. So double coset cayley di-graphs are generalizations of cayley di-graphs.

In the paper entitled "A class of double coset cayley graphs induced by loops"(K. V. Anil, 2011, p. 1073 - 1084), K. V. Anil generalized theorem 1.1 and prove that a class of double coset cayley di-graphs can be induced by loops. It is found that a bigger class of double coset cayley di-graphs can be induced by quasigroups and analogous results are derived concerning properties of the graphs and those of quasigroups. We start with the following definition

Definition 1.2 Let $G$ be a quasigroup, and let $A$ be a subset of $G$. Then $A$ said to be a $\mathcal{R}$ associative (right associative)subset of $G$, if for every $x, y \in G,(x y) A=x(y A)$. This means, if $x, y \in G$ and $a \in A$, then $(x y) a=x\left(y a^{\prime}\right)$ for some $a^{\prime} \in A$. Observe that the $\mathcal{R}$ associative law not only allows to interchange the positions of parenthesis, the left most two elements should be in $G$ and they will be same on both sides, the rightmost element in the left hand side is in $A$ and is changed to another element $a^{\prime} \in A$ as the right most element in the right side. Similarly, we can define $\mathcal{L}$ associative (left associative) subset of $G$.

Lemma 1.3 Let $A$ and $B$ be $\mathcal{R}$ associative ( $\mathcal{L}$ associative) subsets of a quasigroup $G$. Then $A B$ is also $\mathcal{R}$ associative $(\mathcal{L}$ associative).

\section{Main Result}

In this section we generalize the main result of K. V. Anil (International Journal of Algebra, 2011, 5(22), p. 10731084) and prove that a bigger class of double coset Cayley digraphs can be induced by quasigroups. These graphs can be considered as the generalization of double coset cayley di-graphs induced by loops. Moreover analogues results are obtained between the properties of graphs and those of quasigroups. Let $A, B$ and $C$ be subsets of a quasigroup $G$, then we may define the product $A B C$ as follows:

$$
A B C=\{(a b) c: a \in A, b \in B \text { and } c \in C\}
$$

Theorem 2.1 Let $G$ be a quasigroup and let $A$ and $B$ be non empty subquasigroups of $G$ such that $A$ is $\mathcal{L}$ associative and $B$ is $\mathcal{R}$ associative. Let $G /[A, B]$ denote the collection of all double cosets of $A$ and $B$ in $G$. Let $D$ and $D^{*}$ be subsets of $G$ such that $D$ is $\mathcal{L}$ associative and $D^{*}$ is $\mathcal{R}$ associative. Let a and $b$ be fixed elements in $A$ and $B$ respectively. Let

$$
R_{D, D^{*}}=\left\{(A x B, A y B):(a y) b=\left(z_{1} x\right) z_{2} \text { for some } z_{1} \in A D A, z_{2} \in B D^{*} B\right\} .
$$

Then $\left(G /[A, B], R_{D, D^{*}}\right)$ is a graph.

Proof: To see that $\left(G /[A, B], R_{D, D^{*}}\right)$ is a graph, we need only show that $R_{D, D^{*}}$ is well defined. Let $x, x^{\prime}, y$ and $y^{\prime}$ be any four elements of $G$ with $A x B=A x^{\prime} B$ and $A y B=A y^{\prime} B$. Then we have

$$
\left(a x^{\prime}\right) b=\left(a_{1} x\right) b_{1}, \text { for some } a_{1} \in A \text { and } b_{1} \in B
$$

and

$$
\left(a y^{\prime}\right) b=\left(a_{2} y\right) b_{2} \text { for some } a_{2} \in A \text { and } b_{2} \in B \text {. }
$$

If $(A x B, A y B) \in R_{D, D^{*}}$, then

$$
\text { (ay) } b=\left(z_{1} x\right) z_{2} \text { for some } z_{1} \in A D A \text { and } z_{2} \in B D^{*} B \text {. }
$$

Let $z_{1}=\left(a_{3} d_{1}\right) a_{4}$ and $z_{2}=\left(b_{3} d_{2}\right) b_{4}$, where $a_{3}, a_{4} \in A, b_{3}, b_{4} \in B, d_{1} \in D$ and $d_{2} \in D^{*}$. Since $A$ and $B$ are subquasigroups of $G$ there exist unique elements $a_{5} \in A$ and $b_{5} \in B$ such that $a_{5} a=a_{2}$ and $b b_{5}=b_{2}$. Therefore, equation (2) can be written as:

$$
\begin{aligned}
\left(a y^{\prime}\right) b & =\left(\left(a_{5} a\right) y\right)\left(b b_{5}\right) \\
& =\left(a_{6}(a y)\right)\left(b b_{5}\right) \text { for some } a_{6} \in A(\because A \text { is } \mathcal{L} \text { associative }) \\
& =a_{7}\left((a y)\left(b b_{5}\right)\right) \text { for some } a_{7} \in A(\because A \text { is } \mathcal{L} \text { associative }) \\
& =a_{7}\left(((a y) b) b_{6}\right) \text { for some } b_{6} \in A(\because B \text { is } \mathcal{R} \text { associative }) \\
& =a_{7}\left(\left(\left(z_{1} x\right) z_{2}\right) b_{6}\right) \\
& =a_{7}\left(\left(z_{1} x\right)\left(z_{2} b_{7}\right)\right) \text { for some } b_{7} \in B(\because B \text { is } \mathcal{R} \text { associative }) \\
& =a_{7}\left(\left(z_{1} x\right)\left(\left(\left(b_{3} d_{2}\right) b_{4}\right) b_{7}\right)\right) \\
& =a_{7}\left(\left(z_{1} x\right)\left(\left(b_{3} d_{2}\right)\left(b_{4} b_{8}\right)\right) \text { for some } b_{8} \in B(\because B \text { is } \mathcal{R} \text { associative }) .\right.
\end{aligned}
$$


Since $B$ is a subquasigroup of $G$, there exist unique elements $b_{9}, b_{10} \in B$ such that $b_{4} b_{8}=b_{9}$ and $b_{1} b_{10}=b_{3}$. Hence equation (4) can be written as:

$$
\begin{aligned}
\left(a y^{\prime}\right) b & =a_{7}\left(\left(z_{1} x\right)\left(\left(b_{3} d_{2}\right) b_{9}\right)\right. \\
& \left.=a_{7}\left(\left(z_{1} x\right)\left(\left(\left(b_{1} b_{10}\right)\right) d_{2}\right) b_{9}\right)\right) \\
& =a_{7}\left(\left(z_{1} x\right)\left(\left(b_{1}\left(b_{10} d_{3}\right)\right) b_{9}\right)\right) \text { for some } d_{3} \in D^{*}\left(\because D^{*} \text { is } \mathcal{R} \text { associative }\right) \\
& \left.=a_{7}\left(\left(z_{1} x\right)\left(b_{1}\left(b_{10} d_{3}\right) b_{11}\right)\right)\right) \text { for some } b_{11} \in B(\because B \text { is } \mathcal{R} \text { associative }) \\
& =a_{7}\left(\left(z_{1} x\right)\left(b_{1} z_{3}\right)\right) \text { where } z_{3}=\left(b_{10} d_{3}\right) b_{11} \in B D^{*} B . \\
& =a_{7}\left(\left(\left(z_{1} x\right) b_{1}\right) z_{4}\right) \text { for some } z_{4} \in B D^{*} B\left(\because B D^{*} B \text { is } \mathcal{R} \text { associative }\right) \\
& =\left(a_{8}\left(\left(z_{1} x\right) b_{1}\right)\right) z_{4} \text { for some } a_{8} \in A(\because A \text { is } \mathcal{L} \text { associative }) \\
& =\left(\left(a_{9}\left(z_{1} x\right)\right) b_{1}\right) z_{4} \text { for some } a_{9} \in A(\because A \text { is } \mathcal{L} \text { associative }) \\
& =\left(\left(\left(a_{10} z_{1}\right) x\right) b_{1}\right) z_{4} \text { for some } a_{10} \in A(\because A \text { is } \mathcal{L} \text { associative }) \\
& =\left(\left(\left(a_{10}\left(\left(a_{3} d_{1}\right) a_{4}\right)\right) x\right) b_{1}\right) z_{4} \\
& =\left(\left(\left(\left(a_{11}\left(a_{3} d_{1}\right) a_{4}\right)\right) x\right) b_{1}\right) z_{4} \text { for some } a_{11} \in A(\because A \text { is } \mathcal{L} \text { associative }) \\
& =\left(\left(\left(\left(\left(a_{12} a_{3}\right) d_{1}\right) a_{4}\right) x\right) b_{1}\right) z_{4} \text { for some } a_{12} \in A(\because A \text { is } \mathcal{L} \text { associative }) \\
& =\left(\left(\left(\left(a_{13} d_{1}\right) a_{4}\right) x\right) b_{1}\right) z_{4} \text { for some } a_{13} \in A(\because A \text { is a subquasigroup }) .
\end{aligned}
$$

Since $A$ is a subquasigroup of $G$ there exits a unique element $a_{14} \in A$ such that $a_{4}=a_{14} a_{1}$. Hence the above equation can be written as:

$$
\begin{aligned}
\left(a y^{\prime}\right) b & =\left(\left(\left(\left(a_{13} d_{1}\right)\left(a_{14} a_{1}\right)\right) x\right) b_{1}\right) z_{4} \\
& \left.=\left(\left(\left(\left(a_{15} d_{4}\right) a_{14}\right) a_{1}\right) x\right) b_{1}\right) z_{4} \text { for some } a_{15} \in A, d_{4} \in D(\because A D \text { is } \mathcal{L} \text { associative }) \\
& =\left(\left(\left(z_{5} a_{1}\right) x\right) b_{1}\right) z_{4} \text { where } z_{5}=\left(a_{15} d_{4}\right) a_{14} \in A D A \\
& =\left(\left(z_{6}\left(a_{1} x\right)\right) b_{1}\right) z_{4} \text { for some } z_{6} \in A D A(\because A D A \text { is } \mathcal{L} \text { associative }) \\
& =\left(z_{7}\left(\left(a_{1} x\right) b_{1}\right)\right) z_{4} \text { for some } z_{7} \in A D A(\because A D A \text { is } \mathcal{L} \text { associative }) \\
& =\left(z_{7}\left(\left(a x^{\prime}\right) b\right)\right) z_{4} \\
& =\left(\left(z_{8}\left(a x^{\prime}\right)\right) b\right) z_{4} \text { for some } z_{8} \in A D A(\because A D A \text { is } \mathcal{L} \text { associative }) \\
& =\left(\left(\left(z_{9} a\right) x^{\prime}\right) b\right) z_{4} \text { for some } z_{9} \in A D A(\because A D A \text { is } \mathcal{L} \text { associative }) \\
& =\left(\left(\left(\left(\left(a_{16} d_{5}\right) a_{17}\right) a\right) x^{\prime}\right) b\right) z_{4} \text { where } z_{9}=\left(a_{16} d_{5}\right) a_{17} \in A D A \\
& =\left(\left(\left(\left(a_{18}\left(d_{5} a_{17}\right)\right) a\right) x^{\prime}\right) b\right) z_{4} \text { for some } a_{18} \in A(\because A \text { is } \mathcal{L} \text { associative }) \\
& =\left(\left(\left(a_{19}\left(\left(d_{5} a_{17}\right) a\right)\right) x^{\prime}\right) b\right) z_{4} \text { for some } a_{19} \in A(\because A \text { is } \mathcal{L} \text { associative }) \\
& =\left(\left(a_{19}\left(d_{6}\left(a_{17} a\right)\right) x^{\prime}\right) b\right) z_{4} \text { for some } d_{6} \in D(\because D \text { is } \mathcal{L} \text { associative }) \\
& =\left(\left(\left(a_{19}\left(d_{6} a_{20}\right) x^{\prime}\right) b\right) z_{4} \text { where } a_{20}=a_{1} 7 a \in A \text { Since } A\right. \text { is a subquasigroup } \\
& \left.=\left(\left(\left(a_{21} d_{6}\right) a_{20}\right) x^{\prime}\right) b\right) z_{4} \text { for some } a_{21} \in A(\because A \text { is } \mathcal{L} \text { associative }) \\
& =\left(\left(z_{10} x^{\prime}\right) b\right) z_{4} \text { where } z_{10}=\left(a_{21} d_{6}\right) a_{20} \\
& =\left(z_{10} x^{\prime}\right)\left(b z_{11}\right) \text { for some } z_{11} \in B D^{*} B\left(\because B D^{*} B \text { is } \mathcal{R} \text { associative }\right) \\
& =\left(z_{10} x^{\prime}\right)\left(b\left(\left(b_{12} d_{7}\right) b_{13}\right)\right) \text { where } z_{11}=\left(b_{12} d_{7}\right) b_{13} \in B D^{*} B \\
& =\left(z_{10} x^{\prime}\right)\left(\left(b\left(b_{12} d_{7}\right)\right) b_{14}\right) \text { for some } b_{14} \in B(\because B \text { is } \mathcal{R} \text { associative }) \\
& =\left(z_{10} x^{\prime}\right)\left(\left(\left(b b_{12}\right) d_{8}\right) b_{14}\right) \text { for some } d_{8} \in D^{*}\left(\because D^{*} \text { is } \mathcal{R} \text { associative }\right) \\
& =\left(z_{10} x^{\prime}\right)\left(\left(b_{15} d_{8}\right) b_{14}\right) \text { where } b_{15}=b b_{12}(\because B \text { is a quasisubgroup }) \\
& =\left(z_{10} x^{\prime}\right) z_{12} \text { where } z_{12}=\left(b_{15} d_{8}\right) b_{14} .
\end{aligned}
$$

The above equation tells us that $\left(A x^{\prime} B, A y^{\prime} B\right) \in R_{D, D^{*}}$.

Similarly, if $\left(A x^{\prime} B, A y^{\prime} B\right) \in R_{D, D^{*}}$, then one can prove that $(A x B, A y B) \in R_{D, D^{*}}$. Hence $\left(G /[A, B], R_{D, D^{*}}\right)$ is a graph.

We are now ready to define double coset cayley di-graphs induced by quasigroups.

Definition 2.2 The graph defined in theorem 2.1 is called the double coset cayley graphs induced by quasigroups.

\section{Examples}

In this section, we give some interesting examples of double coset cayley graphs induced by quasigroups. 
Let $G=\{0,1,2,3,4,5,6,7\}$. Define a binary operation in $G$ as follows:

\begin{tabular}{l|llllllll}
$*$ & 0 & 1 & 2 & 3 & 4 & 5 & 6 & 7 \\
\hline 0 & 1 & 0 & 3 & 2 & 4 & 5 & 7 & 6 \\
1 & 0 & 1 & 2 & 3 & 5 & 4 & 6 & 7 \\
2 & 2 & 3 & 0 & 1 & 6 & 7 & 5 & 4 \\
3 & 3 & 2 & 1 & 0 & 7 & 6 & 4 & 5 \\
4 & 5 & 4 & 7 & 6 & 1 & 0 & 3 & 2 \\
5 & 4 & 5 & 6 & 7 & 0 & 1 & 2 & 3 \\
6 & 7 & 6 & 4 & 5 & 3 & 2 & 0 & 1 \\
7 & 6 & 7 & 5 & 4 & 2 & 3 & 1 & 0
\end{tabular}

Under this operation $G$ is a quasigroup.

1. Let $A=\{0,1\}, B=\{0,1,2,3\}, D=\{4,5\}$, and $D^{*}=\{6,7\}$. We find that $A$ and $B$ are respectively $\mathcal{L}$ associative and $\mathcal{R}$ associative subquasigroups of $G$. Furthermore, $D$ and $D^{*}$ are respectively $\mathcal{L}$ associative and $\mathcal{R}$ associative subsets of $G$. Take $a=0$ in $A$ and $b=2$ in $B$. On examination we find that

$$
G /[A, B]=\{A 0 B, A 4 B\} \text { and } R_{D, D^{*}}=\{(A 0 B, A 0 B),(A 4 B, A 4 B)\} .
$$

The graphical representation of $\left(G /[A, B], R_{D, D^{*}}\right)$ is shown in figure $1(a)$.

2. Let $A=\{0,1\}, B=\{0,1,2,3\}, D=\{4,5,6,7\}$ and $D^{*}=\{2,3\}$. One can easily verify that $A$ and $B$ are respectively $\mathcal{L}$ associative and $\mathcal{R}$ associative subquasigroups of $G$. Moreover, $D$ and $D^{*}$ are respectively $\mathcal{L}$ associative and $\mathcal{R}$ associative subsets of $G$. Take $a=0$ in $A$ and $b=2$ in $B$. One can easily verify that

$$
G /[A, B]=\{A 0 B, A 4 B\} \text {, and } R_{D, D^{*}}=\{(A 0 B, A 4 B),(A 4 B, A 0 B)\} .
$$

The double coset cayley graph $\left(G /[A, B], R_{D, D^{*}}\right)$ is shown in figure $1(b)$.

3. Let $A=B=\{0,1\}, D=\{4,5,6,7\}$ and $D^{*}=\{2,3\}$. It is not difficult to verify that $A$ and $B$ are both $\mathcal{L}$ and $\mathcal{R}$ associative subquasigroups of $G$. Also $D$ and $D^{*}$ are respectively $\mathcal{L}$ associative and $\mathcal{R}$ associative subsets of $G$. Take $a=0$ in $A$ and $b=2$ in $B$. We find that

$$
\begin{aligned}
G /[A, B]= & \{A 0 B, A 2 B, A 4 B, A 6 B\}, \\
R_{D, D^{*}}= & \{(A 0 B, A 4 B),(A 0 B, A 6 B),(A 2 B, A 4 B),(A 2 B, A 6 B), \\
& (A 4 B, A 0 B),(A 6 B, A 0 B),(A 4 B, A 2 B),(A 6 B, A 2 B)\} .
\end{aligned}
$$

The corresponding double coset cayley graph is shown in figure 1(c).

4. Take the following subsets of $G$ :

$$
A=B=D=\{0,1\} \text { and } D^{*}=\{2,3,4,5,6,7\}
$$

Observe that $A, B$ and $D$ are $\mathcal{L}$ as well as $\mathcal{R}$ associative subquasigroups of $G$ and $D^{*}$ is a right associative subset of $G$. We note that the set of all distinct double cosets of $G$ relative to $A$ and $B$ is

$$
G /[A, B]=\{A 0 B, A 2 B, A 4 B, A 6 B\}
$$

If we take $a=0$ in $A$ and $b=0$ in $B$, it is easy to verify that

$$
\begin{aligned}
R_{D, D^{*}}=\{ & (A 0 B, A 2 B),(A 0 B, A 4 B),(A 0 B, A 6 B),(A 2 B, A 0 B), \\
& (A 2 B, A 4 B),(A 2 B, A 6 B),(A 4 B, A 0 B),(A 4 B, A 2 B) \\
& (A 4 B, A 6 B),(A 6 B, A 0 B),(A 6 B, A 2 B),(A 6 B, A 4 B)\} .
\end{aligned}
$$

A graphical representation of $\left(G /[A, B], R_{D, D^{*}}\right)$ is shown in figure $1(d)$. 


\section{Corollaries}

In this section, we prove some interesting relationship between the properties of $\left(G /[A, B], R_{D, D^{*}}\right)$ and those of quasigroups. Throughout we will suppose that $G$ is a quasigroup, $A$ is a $\mathcal{L}$ associative subquasigroup of $G, B$ is a $\mathcal{R}$ associative subquasigroup of $G, D$ is a $\mathcal{L}$ associative subset of $G, D^{*}$ is a $\mathcal{R}$ associative subset of $G, a$ is a fixed element in $A$ and $b$ is a fixed element in $B$. We begin with the following lemma:

Lemma 4.1 If $z \in A D A, t \in B D^{*} B$, and $x \in G$ then we have

(i) $z=z_{1}$ a for some $z_{1} \in A D A, a z \in A D A$ and $z a \in A D A$ for all $a \in A$.

(ii) $t=b z_{2}$ for some $z_{2} \in B D^{*} B, b t \in B D^{*} D$ and $t b \in B D^{*} B$ for all $b \in B$.

(iii) $(z x) t=\left(z^{*}((a x) b)\right) t^{*}$ for some $z^{*} \in A D A$ and $t^{*} \in B D^{*} B$.

(iv) $(z(a b)) t=z^{*} t^{*}$ for some $z^{*} \in A D A$ and $t^{*} \in B D^{*} B$ and $z t=(u(a b)) v$ for some $u \in A D A, v \in B D^{*} B$.

Proof: (i) Let $z=\left(a_{1} d_{1}\right) a_{2}$ where $a_{1}, a_{2} \in A$ and $d_{1} \in D$. Since $A$ is a subquasigroup, the equation $x a=a_{2}$ has a unique solution in $A$, say $x=a_{3}$. Then $z$ can be re-written as

$$
\begin{aligned}
z & =\left(a_{1} d_{1}\right)\left(a_{3} a\right) \\
& =\left(\left(a_{4} d_{2}\right) a_{3}\right) a \text { for some } a_{4} \in A, d_{2} \in D \text { Since } A D \text { is } \mathcal{L} \text { associative } \\
& =z_{1} a, \text { where } z_{1}=\left(\left(a_{4} d_{2}\right) a_{3}\right) \in A D A .
\end{aligned}
$$

Proofs of the remaining results in (i) are trivial.

(ii) Proof is trivial.

(iii) Observe that, the element $(z x) t$ can be written as:

$$
\begin{aligned}
(z x) t & =\left(\left(z_{1} a\right) x\right) t \text { for some } z_{1} \in A D A \\
& =\left(z_{2}(a x)\right) t \text { for some } z_{2} \in A D A \\
& =\left(z_{2}(a x)\right)\left(b t_{1}\right) \text { for some } t_{1} \in B D^{*} B \\
& =z_{3}\left(((a x) b) t_{2}\right) \text { for some } t_{2} \in B D^{*} B \\
& =\left(z_{4}((a x) b)\right) t_{2} \text { for some } z_{4} \in A D A .
\end{aligned}
$$

(iv) Proof is exactly similar to preceding one.

Let $M$ and $N$ be subsets of a quasigroup $G$ and let $a$ and $b$ be fixed elements in $G$. We will use the following notations:

(1) $[M \mid N]_{a}^{b}=\left\{x \in G:(a x) b=\left(z_{1} x\right) z_{2}\right.$ for some $\left.z_{1} \in M, z_{2} \in N\right\}$.

(2) $M_{a} N_{b}=\left\{x \in G:(a(a b)) b=\left(z_{1} x\right) z_{2}\right.$ for some $\left.z_{1} \in M, z_{2} \in N\right\}$.

(3) $\left[M_{a} \mid N_{b}\right]=\left\{x \in G:(a x) b=z_{1} z_{2}\right.$ for some $\left.z_{1} \in M, z_{2} \in N\right\}$.

(4) $[[M \mid N]]_{a}^{b}=\left\{x \in G:(a x) b=\left(z_{1}\left(z_{2} \ldots\left(z_{n-1}\left(z_{n} t_{n}\right) t_{n-1}\right) t_{n-1} \ldots\right) t_{1}\right.\right.$ for some $\left.z_{i} \in M, t_{i} \in N\right\}$.

(5) $\left[\left[M_{a} N_{b}\right]\right]=\left\{x \in G:(a(a b)) b=\left(z_{1}\left(z_{2} \ldots\left(z_{n-1}\left(z_{n} x t_{n}\right)\right) t_{n-1} \ldots\right) t_{1}\right.\right.$ for some $\left.z_{i} \in M, t_{i} \in N\right\}$.

(6) $[M \mid N]=\left\{z_{1}\left(z_{2} \cdots\left(\left(z_{n-1}\left(z_{n} t_{n}\right)\right) t_{n-1}\right) \cdots t_{2}\right) t_{1}: z_{i} \in M, t_{i} \in N, n=1,2,3, \ldots\right\}$.

Corollary 4.2 The graph $\left(G /[A, B], R_{D, D^{*}}\right)$ is an empty if and only if $D=\emptyset$ or $D^{*}=\emptyset$.

Proof: Observe that $\left(G /[A, B], R_{D, D^{*}}\right)$ is trivial if and only if $R_{D, D^{*}}=\emptyset$. Since $A$ and $B$ are nonempty subquasigroups of $G, D=\emptyset$ or $D^{*}=\emptyset$.

Corollary 4.3 The graph $\left(G /[A, B], R_{D, D^{*}}\right)$ is a reflexive if and only if $G=\left[(A D A) \mid\left(B D^{*} B\right)\right]_{a}^{b}$. Proof: Suppose that $\left(G /[A, B], R_{D, D^{*}}\right)$ is a reflexive and let $x \in G$. Then $(A x B, A x B) \in R_{D, D^{*}}$. This implies that

$$
(a x) b=\left(z_{1} x\right) z_{2} \text { for some } z_{1} \in A D A \text { and } z_{2} \in B D^{*} B .
$$

Therefore, we have $G=\left[A D A \mid B D^{*} B\right]_{a}^{b}$. The converse is trivial. 
Corollary 4.4 If $\left(G /[A, B], R_{D, D^{*}}\right)$ is a symmetric graph, then $\left[(A D A)_{a} \mid\left(B D^{*} B\right)_{b}\right]=(A D A)_{a}\left(B D^{*} B\right)_{b}$.

Proof: First, assume that $\left(G /[A, B], R_{D, D^{*}}\right)$ is symmetric. Observe that

$$
\begin{aligned}
x \in\left[(A D A)_{a} \mid\left(B D^{*} B\right)_{b}\right] & \Leftrightarrow(a x) b=z_{1} z_{2}, \text { for some } z_{1} \in A D A, z_{2} \in B D^{*} B \\
& \Leftrightarrow(a x) b=\left(z_{1}^{*}(a b)\right) z_{2}^{*} \text { for some } z_{1}^{*} \in A D A, z_{2}^{*} \in B D^{*} B \text { by Lemma 4.1) } \\
& \Leftrightarrow(A a b B, A x B) \in R_{D, D^{*}} \\
& \Leftrightarrow(A x B, A a b B) \in R_{D, D^{*}}\left(\text { since } R_{D, D^{*}}\right. \text { is symmetric) } \\
& \Leftrightarrow(a(a b)) b=\left(t_{1} x\right) t_{2} \text { for some } t_{1} \in A D A, t_{2} \in B D^{*} B \\
& \Leftrightarrow x \in(A D A)_{a}\left(B D^{*} B\right)_{b} .
\end{aligned}
$$

This implies that $\left[(A D A)_{a} \mid\left(B D^{*} B\right)_{b}\right]=(A D A)_{a}(B D B)_{b}$.

Corollary $4.5\left(G /[A, B], R_{D, D^{*}}\right)$ is a transitive graph, then $(A D A D A)\left(B D^{*} B D^{*} B\right) \subseteq(A D A)\left(B D^{*} B\right)$.

Proof: Assume that $\left(G /[A, B], R_{D, D^{*}}\right)$ is a transitive graph. Then for all $z_{1}, z_{2} \in A D A, z_{3}, z_{4} \in B D^{*} B$, we have

$$
\begin{aligned}
\left(a\left(z_{1} z_{3}\right)\right) b & \left.=\left(\left(a_{1} z_{1}\right)\right) z_{3}\right) b \text { for some } a_{1} \in A(\because A \text { is } \mathcal{L} \text { associative }) \\
& =\left(z_{5} z_{3}\right) b \text { for some } z_{5} \in B(\text { is by Lemma } 4.1) \\
& =z_{5}\left(z_{3} b_{1}\right) \text { for some } b_{1} \in B(\because B \text { is } \mathcal{R} \text { associative }) \\
& =z_{5} z_{6} \text { for some } z_{6} \in B(\text { by Lemma } 4.1) \\
& \left.=\left(z_{7}(a b)\right) z_{8} \text { for some } z_{7} \in A D A, z_{8} \in B D^{*} B \text { (by Lemma } 4.1\right) .
\end{aligned}
$$

This implies that $\left(A(a b) B, A z_{1} z_{3} B\right) \in R_{D, D^{*}}$. Let $t=z_{1} z_{3}$. Then

$$
\begin{aligned}
\left(a\left(\left(z_{2} t_{1}\right) z_{4}\right)\right) b & =\left(\left(a_{2}\left(z_{2} t_{1}\right)\right) z_{4}\right) b \text { for some } a_{2} \in A(\because A \text { is } \mathcal{L} \text { associative }) \\
& =\left(\left(\left(a_{3} z_{2}\right) t_{1}\right) z_{4}\right) b \text { for some } a_{3} \in A(\because A \text { is } \mathcal{L} \text { associative }) \\
& =\left(\left(z_{9} t_{1}\right) z_{4}\right) b \text { for some } z_{9} \in B(\text { by Lemma } 4.1) \\
& =\left(z_{9} t_{1}\right)\left(z_{4} b_{2}\right) \text { for some } b_{2} \in B(\because B \text { is } \mathcal{R} \text { associative }) \\
& =\left(z_{9} t_{1}\right) z_{10} \text { for some } z_{10} \in B D^{*} B(\text { by Lemma } 4.1) .
\end{aligned}
$$

This implies that $\left(A t_{1} B, A\left(\left(z_{2} t_{1}\right) z_{4} B\right)\right) \in R_{D, D^{*}}$. Since $\left(G /[A, B], R_{D, D^{*}}\right)$ is transitive, we have $\left(A a b B, A\left(\left(z_{2} t_{1}\right) z_{4}\right) B\right) \in R_{D, D^{*}}$. This means that

$$
\left(a\left(z_{2} t_{1}\right) z_{4}\right) b=\left(t_{3}(a b)\right) t_{4} \text { for some } t_{3} \in A D A, t_{4} \in B D^{*} B \text {. }
$$

That is,

$$
\left(z_{11} t_{1}\right) z_{12}=\left(t_{3}(a b)\right) t_{4} \text { for some } z_{11} \in A D A, z_{12} \in B D^{*} B
$$

Equivalently,

$$
(A D A D A)\left(B D^{*} B D^{*} B\right) \subseteq(A D A)\left(B D^{*} B\right) .
$$

Corollary 4.6 If $A D A D A \subseteq A D A$ and $B D^{*} B D^{*} B \subseteq B D^{*} B$, then $\left(G /[A, B], R_{D, D^{*}}\right)$ is a transitive graph.

Proof: Let $A x B, A y B$ and $A z B \in G /[A, B]$ such that $(A x B, A y B) \in R_{D, D^{*}}$ and $(A y B, A z B) \in R_{D, D^{*}}$. Then by the definition of $R_{D, D^{*}}$, we have

$$
\begin{aligned}
& (a y) b=\left(z_{1} x\right) z_{2} \text { for some } z_{1} \in A D A, z_{2} \in B D^{*} B \\
& (a z) b=\left(z_{3} y\right) z_{4} \text { for some } z_{3} \in A D A, z_{4} \in B D^{*} B .
\end{aligned}
$$

Equation (6) can be written as:

$$
\begin{aligned}
(a z) b & =\left(\left(z_{5} a\right) y\right)\left(b z_{6}\right) \text { for some } z_{5} \in A D A, z_{6} \in B D^{*} B \text { (by Lemma 4.1) } \\
& =\left(\left(z_{6}(a y)\right) b\right) z_{7} \text { for some } z_{7} \in B D^{*} B\left(\because B D^{*} B \text { is } \mathcal{R} \text { associative }\right) \\
& =\left(z_{8}((a y) b)\right) z_{7} \text { for some } z_{8} \in A D A(\because A D A \text { is } \mathcal{L} \text { associative }) .
\end{aligned}
$$


Using equation (5) in equation (7), we get

$$
\begin{aligned}
(a z) b & =\left(z_{8}\left(z_{1} x\right) z_{2}\right) z_{7} \\
& =\left(\left(z_{9}\left(z_{1} x\right)\right) z_{2}\right) z_{7} \text { for some } z_{9} \in A D A(\because A D A \text { is } \mathcal{L} \text { associative }) \\
& =\left(\left(\left(z_{10} z_{1}\right) x\right) z_{2}\right) z_{7} \text { for some } z_{10} \in A D A(\because A D A \text { is } \mathcal{L} \text { associative }) \\
& =\left(\left(t_{1} x\right) z_{2}\right) z_{7} \text { where } t_{1}=z_{10} z_{1} \in(A D A)(A D A) \\
& =\left(t_{1} x\right)\left(z_{2} z_{11}\right) \text { for some } z_{12} \in B D^{*} B\left(\because B D^{*} B \text { is } \mathcal{R} \text { associative }\right) \\
& =\left(t_{1} x\right) t_{2} \text { where } t_{2}=z_{2} z_{11} \in\left(B D^{*} B\right)\left(B D^{*} B\right) .
\end{aligned}
$$

Since $(A D A)(A D A)=A D A D A \subseteq A D A$ and $\left(B D^{*} B\right)\left(B D^{*} B\right)=B D^{*} B D^{*} B \subseteq B D^{*} B$, equation (8) implies that $(A x B, A z B) \in$ $R_{D, D^{*}}$. Hence $\left(G /[A, B], R_{D, D^{*}}\right)$ is a transitive graph.

Corollary 4.7 If $\left(G /[A, B], R_{D, D^{*}}\right)$ is a complete graph, then $G=\left[(A D A)_{a} \mid\left(B D^{*} B\right)_{b}\right]$.

Proof: Assume that $\left(G /[A, B], R_{D, D^{*}}\right)$ is a complete graph and let $x \in G$. Then $(A(a b) B, A x B) \in R_{D, D^{*}}$. This implies that $(a x) b=\left(z_{1}(a b)\right) z_{2}$, for some $z_{1} \in A D A$ and $z_{2} \in B D^{*} B$. That is, $(a x) b=z_{1}^{*} z_{2}^{*}$, for some $z_{1}^{*} \in A D A$ and $z_{2}^{*} \in B D^{*} B$. Equivalently, $x \in\left[(A D A)_{a} \mid\left(B D^{*} B\right)_{b}\right]$. Since $x$ is an arbitrary element of $G$,

$$
G=\left[(A D A)_{a} \mid\left(B D^{*} B\right)_{b}\right]
$$

Corollary 4.8 If $\left(G /[A, B], R_{D, D^{*}}\right)$ is connected, then $G=\left[\left[A D A \mid B D^{*} B\right]\right]_{a}^{b}$.

Proof: Suppose that $\left(G /[A, B], R_{D, D^{*}}\right)$ is connected and let $x \in G$. Then there is a path from $A B$ to $A x B$, say:

$$
\left(A(a b) B, A x_{1} B, A x_{2} B, \cdots, A x_{n} B, A x B\right)
$$

Then we have the following:

$$
\begin{aligned}
& \left(a x_{1}\right) b=\left(z_{1}(a b)\right) t_{1} \text { for some } z_{1} \in A D A \text { and } t_{1} \in B D^{*} B \\
& =z_{1}^{*} t_{1}^{*} \text { for some } z_{1}^{*} \in A D A \text { and } t_{1}^{*} \in B D^{*} B \quad \text { (by Lemma 4.1), } \\
& \begin{aligned}
\left(a x_{2}\right) b & =\left(z_{2} x_{1}\right) t_{2} \text { for some } z_{2} \in A D A \text { and } t_{2} \in B D^{*} B \\
& =\left(\left(\left(z_{3} a\right) x_{1}\right)\left(b t_{3}\right)\right) \text { for some } z_{3} \in A D A \text { and } t_{3} \in B D^{*} B \\
& =\left(z_{4}\left(\left(a x_{1}\right) b\right)\right) t_{4} \text { for some } z_{4} \in A D A \text { and } t_{4} \in B D^{*} B \\
& \left.=\left(z_{4}\left(z_{1}^{*} t_{1}^{*}\right)\right) t_{4} \quad \text { (by equation }(9)\right)
\end{aligned}
\end{aligned}
$$

If we continue, we obtain

$$
\begin{aligned}
(a x) b & =\left(z_{n+1} x_{n}\right) t_{n+1} \\
& \left.=\left(z_{n+1}^{*}\left(\left(a x_{n}\right) b\right)\right) t_{n+1}^{*} \text { for some } z_{n+1}^{*} \in A D A, t_{n+1}^{*} \in B D^{*} B\right) . \\
& =\left(z_{n+1}^{*}\left(\left(z_{n} x_{n-1}\right) t_{n}\right)\right) t_{n+1}^{*}=\cdots=\left(z_{n+1}^{*}\left(\cdots\left(z_{2}^{*}\left(z_{1}^{*} t_{1}^{*}\right)\right) t_{2}^{*} \cdots\right)\right) t_{n+1}^{*} .
\end{aligned}
$$

Equivalently,

$$
G=\left[\left[A D A \mid B D^{*} B\right]\right]_{a}^{b} .
$$

Corollary 4.9 If $\left(G /[A, B], R_{D, D^{*}}\right)$ is locally connected, then $\left[A D A \mid B D^{*} B\right]=\left[\left[(A D A)_{a}\left(B D^{*} B\right)_{b}\right]\right]$.

Proof: Assume that $\left(G /[A, B], R_{D, D^{*}}\right)$ is locally connected. Let $x \in\left[A D A \mid B D^{*} B\right]$. Then

$$
x=\left(z_{1}\left(z_{2} \ldots\left(z_{n-1}\left(z_{n} t_{n}\right)\right) t_{n-1} \ldots t_{2}\right) t_{1}\right.
$$

for some $z_{i} \in A D A$ and $t_{i} \in B D^{*} B$. Let

$$
x_{1}=z_{n} t_{n}, x_{2}=\left(z_{n-1} x_{1}\right) t_{n-1}, \ldots, x_{n}=\left(z_{1} x_{n-1}\right) t_{1}
$$

Using Lemma 4.1, the above equation can be re-written as:

$$
\left(a x_{1}\right) b=\left(z_{n}^{*}(a b)\right) t_{n}^{*},\left(a x_{2}\right) b=\left(z_{n-1}^{*}(a b) x_{1}^{*}\right) t_{n-1}^{*}, \ldots,\left(a x_{n}^{*}\right) b=\left(z_{1}^{*}(a b) x_{n-1}^{*}\right) t_{1}^{*} .
$$


for some $z_{i}^{*} \in A D A$ and $t_{i}^{*} \in B D^{*} B$. Then $\left(A(a b) B, A x_{1} B, \ldots, A x_{n} B, A x_{n} B\right)$ is a path from $A B$ to $A x B$. Since $\left(G /[A, B], R_{D, D^{*}}\right)$ is locally connected, there exits a path from $A x B$ to $A B$, say:

$$
\left(A x B, A y_{1} B, \ldots, A y_{m} B, A a b B\right)
$$

This implies that $x \in\left[\left[(A D A)_{a}\left(B D^{*} B\right)_{b}\right]\right]$. Hence $\left[A D A \mid B D^{*} B\right] \subseteq\left[\left[(A D A)_{a}\left(B D^{*} B\right)_{b}\right]\right]$. Similarly, $\left[\left[(A D A)_{a}\left(B D^{*} B\right)_{b}\right]\right] \subseteq$ $\left[A D A \mid B D^{*} B\right]$.

Corollary 4.10 If $\left(G /[A, B], R_{D, D^{*}}\right)$ is semi connected, then $G=\left[\left[A D A \mid B D^{*} B\right]\right]_{a}^{b} \cup\left[\left[(A D A)_{a}\left(B D^{*} B\right)_{b}\right]\right]$.

Proof: Assume that $\left(G /[A, B], R_{D, D^{*}}\right)$ is semi connected and let $x \in G$. Then there exits a path from $A B$ to $A x B$, say

$$
\left(A(a b) B, A x_{1} B, \ldots, A x_{n} B, A x B\right)
$$

or a path from $A x B$ to $A a b B$, say

$$
\left(A x B, A y_{1} B, \ldots, A y_{m} B, A(a b) B\right)
$$

This implies that

$$
x \in\left[\left[A D A \mid B D^{*} B\right]\right]_{a}^{b} \cup\left[\left[(A D A)_{a}\left(B D^{*} B\right)_{b}\right]\right] .
$$

Since $x$ is arbitrary, it follows that

$$
G=\left[[ A D A | B D ^ { * } B ] \left[{ }_{a}^{b} \cup\left[\left[(A D A)_{a}\left(B D^{*} B\right)_{b}\right]\right] .\right.\right.
$$

Corollary 4.11 $\left(G /[A, B], R_{D, D^{*}}\right)$ is a hasse- diagram, if and only if $(A D A)^{n} \cap(A D A)=\emptyset$ or $\left(B D^{*} B\right)^{n} \cap\left(B D^{*} B\right)=\emptyset, n \geq 2$.

Proof: First, assume that $\left(G /[A, B], R_{D, D^{*}}\right)$ is a hasse- diagram. Then for any vertices $A x_{0} B, A x_{1} B, \ldots, A x_{n} B \in G /[A, B]$ with $\left(A x_{i} B, A x_{i+1} B\right) \in R_{D, D^{*}}$ for all $i=0,1,2, \ldots, n-1$ implies that $\left(A x_{0} B, A x_{n} B\right) \notin R_{D, D^{*}}$. Observe that $\left(A x_{i} B, A x_{i+1} B\right) \in$ $R_{D, D^{*}}$ for all $i=0,1,2, \ldots, n-1$ implies that

$$
\left(a x_{i+1}\right) b=\left(z_{i} x_{0}\right) t_{i} \text { for some } z_{i} \in A D A \text { and } t_{i} \in B D^{*} B \text { for } i=0,1,2, \ldots, n-1 .
$$

Putting $n=0,1,2, \ldots(n-1)$ successively in equation (10), we get

$$
\begin{aligned}
\left(a x_{1}\right) b & =\left(z_{1} x_{0}\right) t_{1} \\
\left(a x_{2}\right) b & =\left(z_{2} x_{1}\right) t_{2} \\
\left(a x_{3}\right) b & =\left(z_{3} x_{2}\right) t_{3} \\
\vdots & \\
\left(a x_{n}\right) b & =\left(z_{n} x_{n-1}\right) t_{n}
\end{aligned}
$$

Using Lemma 4.1, above equations can be re-written as:

$$
\begin{aligned}
\left(a x_{2}\right) b & =\left(u_{1}\left(\left(a x_{1}\right) b\right)\right) v_{1} \text { for some } u_{1} \in A D A \text { and } v_{1} \in B D^{*} B \\
& =\left(u_{1}\left(\left(z_{1} x_{0}\right) t_{1}\right)\right) v_{1} \\
& =\left(\left(u_{2}\left(z_{1} x_{0}\right)\right) t_{1}\right) v_{1} \text { for some } u_{2} \in A D A \text { and } v_{1} \in B D^{*} B \\
& \left.=\left(\left(u_{3} z_{1}\right) x_{0}\right) t_{1}\right) v_{1} \text { for some } z_{1} i n A D A \text { and } v_{1} \in B D^{*} B \\
& =\left(\left(u_{3} z_{1}\right) x_{0}\right)\left(t_{1} v_{2}\right) \\
& =\left(r_{1} x_{0}\right) s_{1} \text { where } r_{1}=u_{3} z_{1} \in(A D A)^{2} \text { and } s_{1}=t_{1} v_{1} \in\left(B D^{*} B\right)^{2} .
\end{aligned}
$$

Similarly, $\left(a x_{3}\right) b=\left(r_{2} x_{0}\right) s_{2}$ where $r_{2} \in(A D A)^{3}$ and $s_{2} \in\left(B D^{*} B\right)^{3}$ Proceeding like this, we get $\left(a x_{n}\right) b=$ $r_{n} x_{0} s_{n}$ for some $r_{n} \in(A D A)^{n}$ and $s_{n} \in\left(B D^{*} B\right)^{n}$ Since $\left(A x_{0} B, A x_{n} B\right) \notin R_{D, D^{*}}$, therefore

$$
(A D A)^{n} \cap(A D A)=\emptyset \text { or }\left(B D^{*} B\right)^{n} \cap\left(B D^{*} B\right)=\emptyset .
$$

Conversely, assume that

$$
(A D A)^{n} \cap(A D A)=\emptyset \text { or }\left(B D^{*} B\right)^{n} \cap\left(B D^{*} B\right)=\emptyset, n \geq 2 .
$$

We will show that $\left(G /[A, B], R_{D, D^{*}}\right)$ is a hasse-diagram. Let

$$
A x_{0} B, A x_{1} B, \ldots, A x_{n} B
$$


be any $(n+1)$ elements of $G /[A, B]$ with $n \geq 2$, and $\left(A x_{i} B, A_{x i+1} B\right) \in R_{D, D^{*}}$ for all $i=0,1,2, \ldots, n-1$. Then we have

$$
\left(a x_{n}\right) b=\left(z x_{0}\right) t \text { for some } z \in(A D A)^{n} \text { and } t \in\left(B D^{*} B\right)^{n}
$$

Since $(A D A)^{n} \cap(A D A)=\emptyset$ or $\left(B D^{*} B\right)^{n} \cap\left(B D^{*} B\right)=\emptyset,\left(A x_{0} B, A x_{n} B\right) \notin R_{D, D^{*}}$. Hence $\left(G /[A, B], R_{D, D^{*}}\right)$ is a hasse-diagram.

\section{Open Problem}

In this paper we have proved that a class of double coset cayley graphs could be induced by quasigroups. It is well known that all cayley graphs induced by groups are vertex transitive graphs. One can naturally ask the question: are the double coset cayley graphs induced by quasigroups vertex transitive? So we conclude this section with the following problem:

Prove or disprove that $\left(G /[A, B], R_{D, D^{*}}\right)$ is vertex transitive.

\section{Acknowledgment}

The authors would like to thank the referee for his valuable suggestions which has helped us to improve this paper.

\section{References}

B. Alspach, \& C. Q. Zhang. (1989). Hamilton cycles in cubic Cayley graphs on dihedral groups. Ars Combin., 28, 101-108.

B. Alspach, S. Locke, \& D. Witte. (1990). The Hamilton spaces of Cayley graphs on abelian groups. Discrete Math., 82, 113-126. http://dx.doi.org/10.1016/0012-365X(90)90319-D

B. Alspach, \& Y. Qin. (2001). Hamilton-Connected Cayley graphs on Hamiltonian groups. Europ. J. Combin., 22, 777-787. http://dx.doi.org/10.1006/eujc.2001.0456

C. Godsil, \& R. Gordon. (2001). Algebraic Graph Theory, Graduate Texts in Mathematics. New York: Springer-Verlag.

D. Witte. (1982). On Hamiltonian circuits in Cayley diagrams. Discrete Math., 38, 99-108. http://dx.doi.org/10.1016/0012-365X(82)90174-1

D. Witte. (1986). Cayley digraphs of prime-power order are Hamiltonian. J. Combin. Theory Ser. B, 40, $107-112$. http://dx.doi.org/10.1016/0095-8956(86)90068-7

D. Witte, \& K. Keating. (1985). On Hamilton cycles in Cayley graphs in groups with cyclic commutator subgroup. Ann. Discrete Math., 27, 89-102.

E. Dobson. (2006). Automorphism groups of Metacirculant graphs of order a product of two distinct primes. Combinatorics, Probability and Computing, 15, 105-130. http://dx.doi.org/10.1017/S0963548305007066

G. Sabidussi. (1958). On a class of fixed-point-free graphs. Proc. Amer. Math. Soc., 9, $800-804$. http://dx.doi.org/10.1090/S0002-9939-1958-0097068-7

H. B. Richard. (1971). A survey of Binary Systems. New York: Springer-Verlag.

J. B. Fraleigh. (2008). A First course in abstract algebra. Pearson Education.

K. V. Anil. (2011). Double Coset Cayley Digraphs. International Journal of Algebra, 5 (15), 747-753.

K. V. Anil. (2011). A Class of Double Coset Cayley Digraphs induced by Loops. International Journal of Algebra, 5 (22), 1073-1084.

S. B. Akers, \& B. Krishamurthy. (1989). A group theoritic model for symmetric interconnection networks. IEEE Trans. comput., 38, 555-556. http://dx.doi.org/10.1109/12.21148

S. J. Curran, \& J. A. Gallian. (1996). Hamiltonian cycles and paths in Cayley graphs and digraphs - A survey. Discrete Math., 156, 1-18. http://dx.doi.org/10.1016/0012-365X(95)00072-5

S. Lakshmivarahan, J. Jwo, \& S. K. Dhall. (1993). Symmetry in interconnection networks based on Cayley graphs of permutation groups - A survey. Parallel Comput., 19, 361-407. http://dx.doi.org/10.1016/0167-8191(93)90054-O 


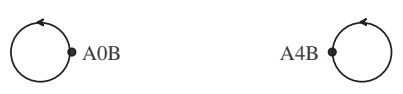

(a)

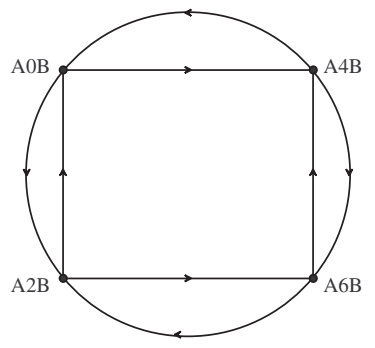

(c)

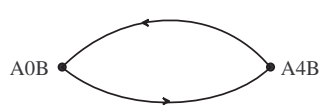

(b)

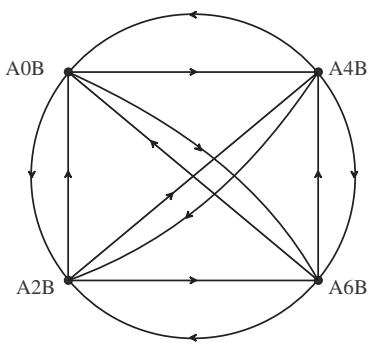

(d)

Figure 1. The Double Coset Cayley di-graphs 